\title{
Effect of Light Quality and Microbiological Inoculum on Geranium (Pelargonium zonale L.) Gas Exchange Parameters
}

\author{
Anita SCHROETER-ZAKRZEWSKA ${ }^{1 *}$, Klaudia BOROWIAK ${ }^{2}$, \\ Agnieszka WOLNA-MARUWKA ${ }^{3}$
}

\author{
IPoznańUniversity ofLife Sciences,DepartmentofOmamental Plants, Dabrowskiego 159, 60-594Poznań,Poland; anitazak@up.poznan.pl('correspondingauthor) \\ ${ }^{2}$ Poznań University of Life Sciences, Department of Ecology andEnvironmental Protection, Piatkouska94C,60-649Poznain, Poland; klaudine@up.poznam.pl \\ ${ }_{3}^{3}$ Poznań University of Life Sciences, DepartmentofGeneral and EnvirommentalMicrobiology, Szydtowska 50, 60-656 Poznań, Poland; amarnwka@interiapl
}

\begin{abstract}
Geranium plants were cultivated in a growth chamber with different light quality and microorganism inoculation conditions. The long-term effect of irradiance quality (LED and fluorescent) as well as two types of microorganism treatments on photosynthetic activity parameters was examined. The maximum reached values of net photosynthesis rate $\left(P_{\mathrm{N}}\right)$, stomatal conductance $\left(g_{\mathrm{s}}\right)$, transpiration rate $(E)$ and intercellular $\mathrm{CO}_{2}$ concentration $\left(C_{\mathrm{i}}\right)$ were afterwards measured in plants cultivated under six colours of light - white, white-blue, blue, red, red-blue and green - emitted by two types of lamp: LED and fluorescent. Two types of microorganism treatments were used: $\mathrm{BAF}_{1}$ created in the Department of General and Environmental Microbiology and the commonly used Effective Microorganisms (EM) biopreparation. A highest level of $P_{\mathrm{N}}$

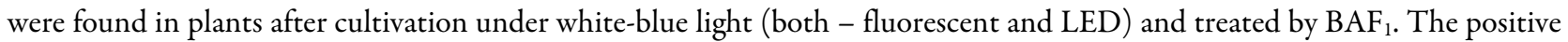
effect of EM was only noted in the case of plants cultivated under blue-red fluorescent light. Considering plants without microbial inoculation the highest levels were recorded in plants under red and blue light. The comparison of the effect of light quality revealed that in all colours a higher or similar level of net photosynthetic rate was noted in plants under LEDs. Mostly the higher photosynthetic activity was connected with higher stomatal opening as well as with higher transpiration rate, which is especially true for plants cultivated under red and blue LED light.
\end{abstract}

Keywords: fluorescent, geranium, Light Emitting Diode (LED), microbiological inoculation, photosynthetic activity

\section{Introduction}

Pelargonium zonale for years has taken a leading position in the rankings of popularity among balcony plants. In recent years there is a marked shift in the retail outlets where these plants are typically bought. They are purchased not only at flower shops and garden centres, but also in supermarkets and discount hypermarkets. These retail outlets provide inferior lighting and temperature conditions, far from those considered optimal for this species. Inappropriate storage conditions result in the deterioration of quality and health condition of plants, leading as a consequence to limited interest of consumers and thus poor sales. As shown in the study by Jerzy et al. (2011), the post-harvest life of Chrysanthemum $\times$ grandiflorum may be extended when plants are kept under lamps emitting different light colours. Fluorescent lamps are used in laboratories to provide lighting for plants. However, due to their high energy costs, cheaper sources, which at the same time emit light more advantageous for plants, are sought. In recent years light emitting diodes (LED) have become increasingly popular and they are considered the lighting of the future (Morrow, 2008; Reinders, 2008). Thanks to their special design, heat is carried away by radiators; as a result, the lamps do not become hot and may be placed directly over plants.

Many investigations have studied the various effects of light quality on photosynthesis. Sæbø et al. (1995) found that red light enhanced starch accumulation during photosynthesis. There is also information about light quality and chlorophyll content, especially and effect of red colour light (Tanaka et al., 1998). However, blue light can also influence photosynthesis due to its effect on chloroplast development and stomata opening (Senger, 1982). There is also a suggestion that the plant response to blue light is stronger due to the presence of blue-light receptors - phototropins (Wu, 2008). Some investigators have revealed that a stronger effect of blue light on stomatal opening is connected with another receptor, zeaxanthin (Zeiger et al., 2002). Light mediates the stomatal opening through regulation of plasma membrane (PM) $\mathrm{H}^{+}$-ATPase activity. The enzyme phosphatase 1 has been found to play an important role in the case of blue light-induced signalling in guard cells (Wu, 2008). It is known that red and blue light are the most important in photomorphogenesis (Voskresenskaya, 1987). Moreover, investigations conducted by Kim et al. (2004a) and Matsuda et al. 
26

(2008) revealed that additional blue and red light promote net photosynthetic rate $\left(P_{\mathrm{N}}\right)$ and subsequently plant growth. Red and blue light affects stomatal conductance due to the presence of chloroplasts in guard cells in many plant species. Also a regulatory role of green light has been found (Golovatskaya, 2005).

Investigations also revealed a strong connection between light quality and photosynthetic activity as well as stomatal conductance of many horticultural plant species. A higher photosynthetic activity level was found for Euphorbia pulcherrima, Plectranthus scutellarioides and Lactuca sativa in plants cultivated under LEDs in comparison to plants cultivated under high pressure sodium (HPS) lamps (Domurath et al., 2012). Moreover, the effect of lamp colour was also examined, and a higher net photosynthetic rate was noted in cherry tomato seedlings cultivated under LEDs with red-blue and red-blue-green light (Liu et al, 2011, Xiaoying et al., 2012) or blue and red-green-blue in the case of orchids (Lee $e t$ al., 2011). Monochromatic light has been found to decrease photosynthesis in comparison to combination of red-blue one in many plant species (Goins et al., 1997; Sabzalian et al.,2014).

The application of microbiological preparations in plant cultivation may bring various benefits. As reported by Stielow (2003), the introduction of the Effective Microorganisms (EM) inoculation to the culture medium may, among other things, reduce putrefaction processes, enhance drought resistance, inhibit development of plant pathogens, and cause more abundant flowering. The above factors are essential during plant storage
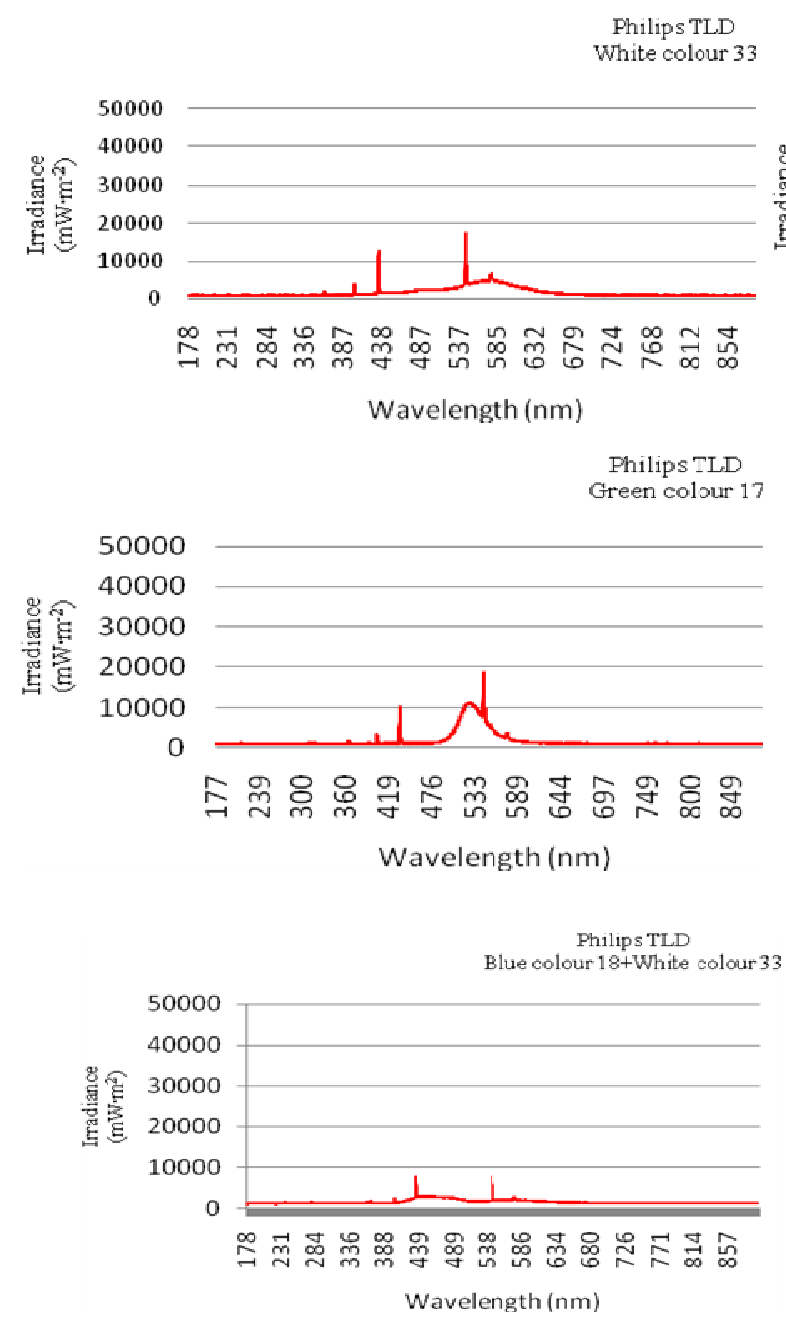

Fig. 1. Spectral characteristic of the fluorescent lamps without access to natural light. Moreover in presented study two types of microbial inoculation were compared. The first one is commonly known EM, while the second is unique combination of bacteria, actinomycetes and fungi, here called $B A F_{1}$. The content of BAF1 includes not only rhyzospheric microorganisms, but also endophytes occurring on plant root surface. Hence the plant response, such as nutrient uptake, growth and photosynthetic activity can be different than to EM (Wolna-Maruwka et al., 2014, 2015).

Microorganisms can also secrete substances (antibiotics, siderophores) which are toxic against to plant pathogens improving the condition and health of plants (Marschner,2007).

Microorganisms contribute to nitrogen and phosphorus uptake (Borkowska, 2002; Martin et al., 2001; Nowak, 2004; Smith and Read, 2008). Higher levels of nutrient sorption are connected with increased enzyme activity (e.g. nitrate reductase, phosphatase, glutamate dehydrogenase, glutamine synthetase). This also leads to increased photosynthesis levels and maintenance of the physiological balance, resulting in elevated plant biomass growth (Martin and Botton, 1993; Smith and Read, 2008).

Simultaneous plant cultivation with certain light quality conditions and microorganism inoculation can result in favourable photosynthesis levels for certain growth stages. Hence a presented work would have two types of aims, one practical - to allow a good photosynthesis level when plants are kept under suboptimal conditions during storage - shops, supermarkets, and maybe indicate
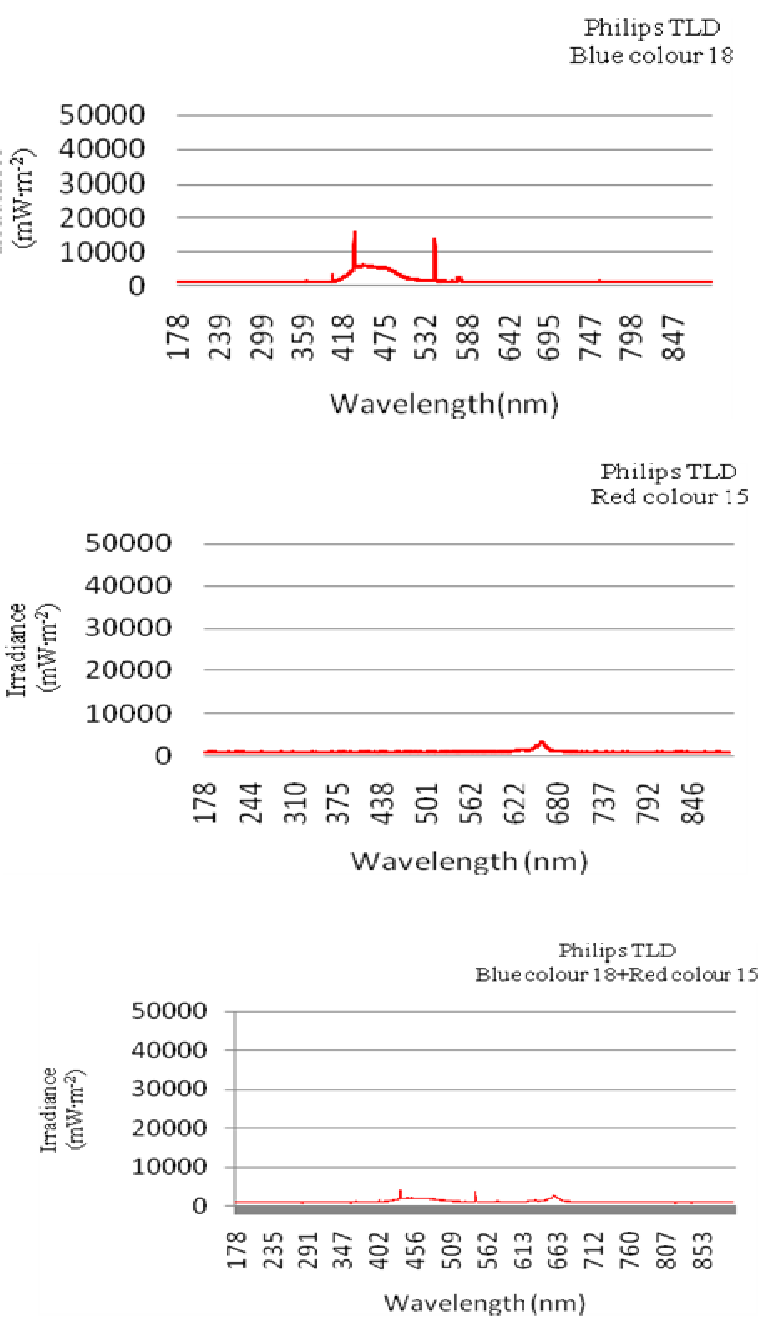
which lamp and microorganisms allow to prolong storage without negative effects on future photosynthesis of plants and in a consequence to flowering. While the scientific aim would focused on effect of several week exposure to various light and microorganisms conditions to photosynthesis system adaptation. The detailed aims of the present study were as follows: (i) to determine the effect of different light quality of exposed plants for several weeks on photosynthesis level; (ii) to examine whether microbiological inoculation influences this process; (iii) to find the proper combination of investigated factors.

\section{Materials and Methods}

The experiment was established in 2012-2013 in a growth chamber at artificial light and under controlled climate conditions. Rooted cuttings of Pelargonium zonale 'Tamara' were planted in pots of $12 \mathrm{~cm}$ diameter into a peat substrate of 5.5-6.0 $\mathrm{pH}$ and supplemented with a slowly released multi-component fertiliser Osmocote 5-6 M (3 g.dm $\left.{ }^{-3}\right)$.

The plants were inoculated with the $\mathrm{BAF}_{1}$ and $\mathrm{EM}$ biopreparations, which were applied onto leaves and into the soil. The preparation was diluted in tap water with the concentration $1: 100$. EM is a microbiological inoculum composed mainly of lactic acid and photosynthetic bacteria, yeasts and actinomycetes (Higa, 1991). The microbiological inoculum $\mathrm{BAF}_{1}$ (BacteriaActinomycetes-Fungi) used in the study was designed at the Department of General and Environmental Microbiology. The biopreparation consisted of 15 strains of bacteria, 5 of actinomycetes isolated from mature compost prepared from plant
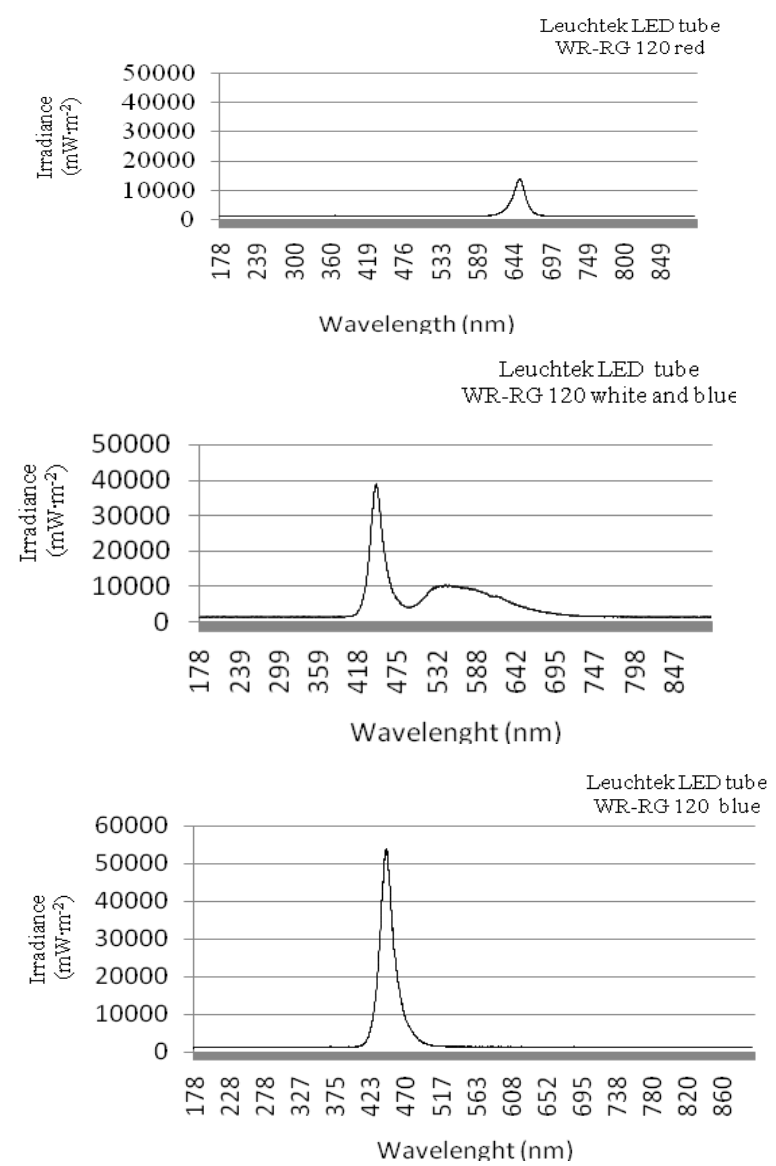

Fig. 2. Spectral characteristic of the LED lamps residues and sewage sludge as well as 4 strains of Trichoderma harzianum fungus derived from the collection of the Institute of Plant Genetics in Poznan. These strains were examined from the point of view of their proteolytic and cellulolytic activities. One millilitre of the employed biopreparation contained $1.55 \cdot 10^{6}$ (colony forming units) cfu of bacteria, $1.99 \cdot 10^{3}$ cfu of actinomycetes and $0.98 \cdot 10^{2}$ cfu of fungi.

The experiment was started at the stage of microscopic development of inflorescence buds, on the day when the buds were still green and closed, but they were ready to change colour and to continue their further development. The height of plants was 30 $\mathrm{cm}$. Pots with plants were placed on shelves in the growth chamber (earlier, the plants had been grown in a greenhouse). Four replicates were conducted according to each microorganisms combinations under each lamp type. The experiment was performed using two types of lamps: fluorescent (PHILIPS TLD) and LED Tube (LEUCHTEK) emitting white, green, red, blue, red-blue and white-blue colours of light. Quantum irradiance (PPFD) was $35 \mu \mathrm{mol} \cdot \mathrm{m}^{-2} \mathrm{~s}^{-1}$ and was measured by means of Optel phytophotometer FR-10 (Sonopan, Poland) at the top of the plants by changing the distance from each light source. Air temperature in the growth chamber was maintained at a constant level of $20 \pm 1{ }^{\circ} \mathrm{C}$ and air humidity was $65-70 \%$. Day length lasted 12 hours. Spectral characteristics of the two types of lamps determined with a spectroradiometer (USB 4000) are shown in Figures 1-2.

The handheld photosynthesis system Ci 340aa (CID BIOSCIENCE Inc., Camas, USA) was used to evaluate net photosynthetic rate $\left(P_{\mathrm{N}}\right)$, stomatal conductance $\left(g_{\mathrm{s}}\right)$, transpiration

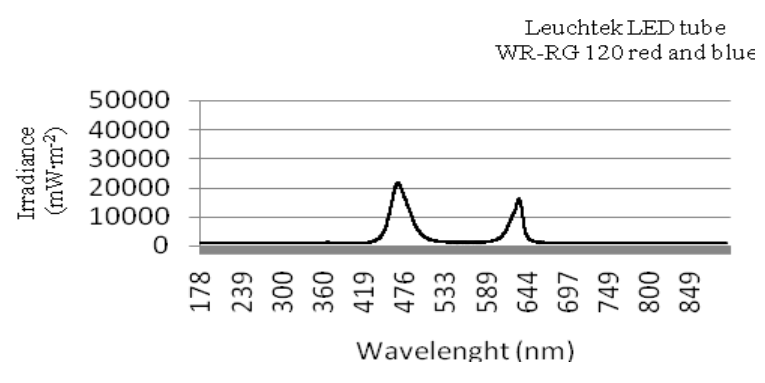

Leuchtek LED tube WR-RG 120 white

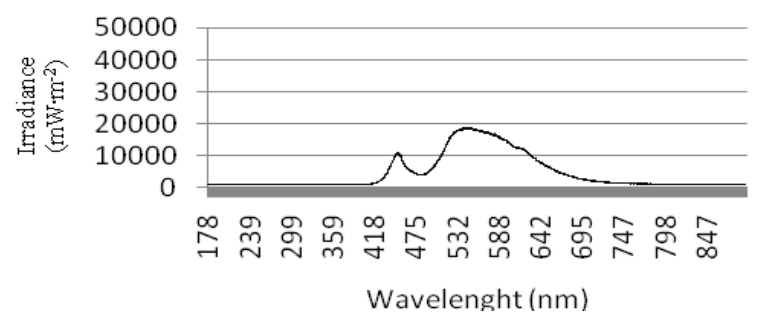

Leuchtek LED tube

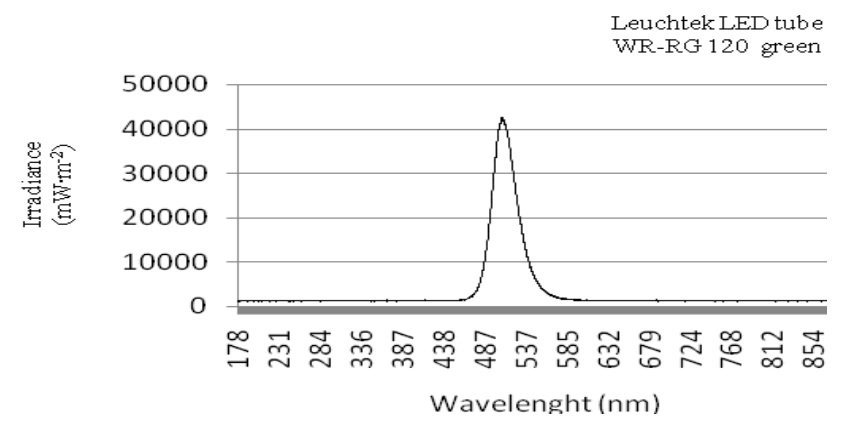


rate $(E)$ and intercellular $\mathrm{CO}_{2}$ concentration $\left(C_{\mathrm{i}}\right)$. For these purposes, constant conditions of measurements in the leaf chamber were maintained: $\mathrm{CO}_{2}$ inflow concentration $(350 \mu \mathrm{mol}$ $\left.\left(\mathrm{CO}_{2}\right) \mathrm{mol}^{-1}\right)$, photosynthetic photon flux density (PPFD) 1000 umol (photon) $\mathrm{m}^{-2} \mathrm{~s}^{-1}$, chamber temperature $23^{\circ} \mathrm{C}$, relative humidity $40 \pm 3 \%$.

The experiment was established in two culture cycles. Results of measurement given as mean from two years of research. The data were analysed with the statistical software STATISTICA 9.1. Results were analysed with a factorial $A N O V A$ with "lamp type", "colour of light" and "type of microbiological inoculation" fixed factors. Tukey's test was employed to analyse differences between measured parameters. A graphical presentation of Tukey's test results is provided in the present study as well as table data with statistical error values.

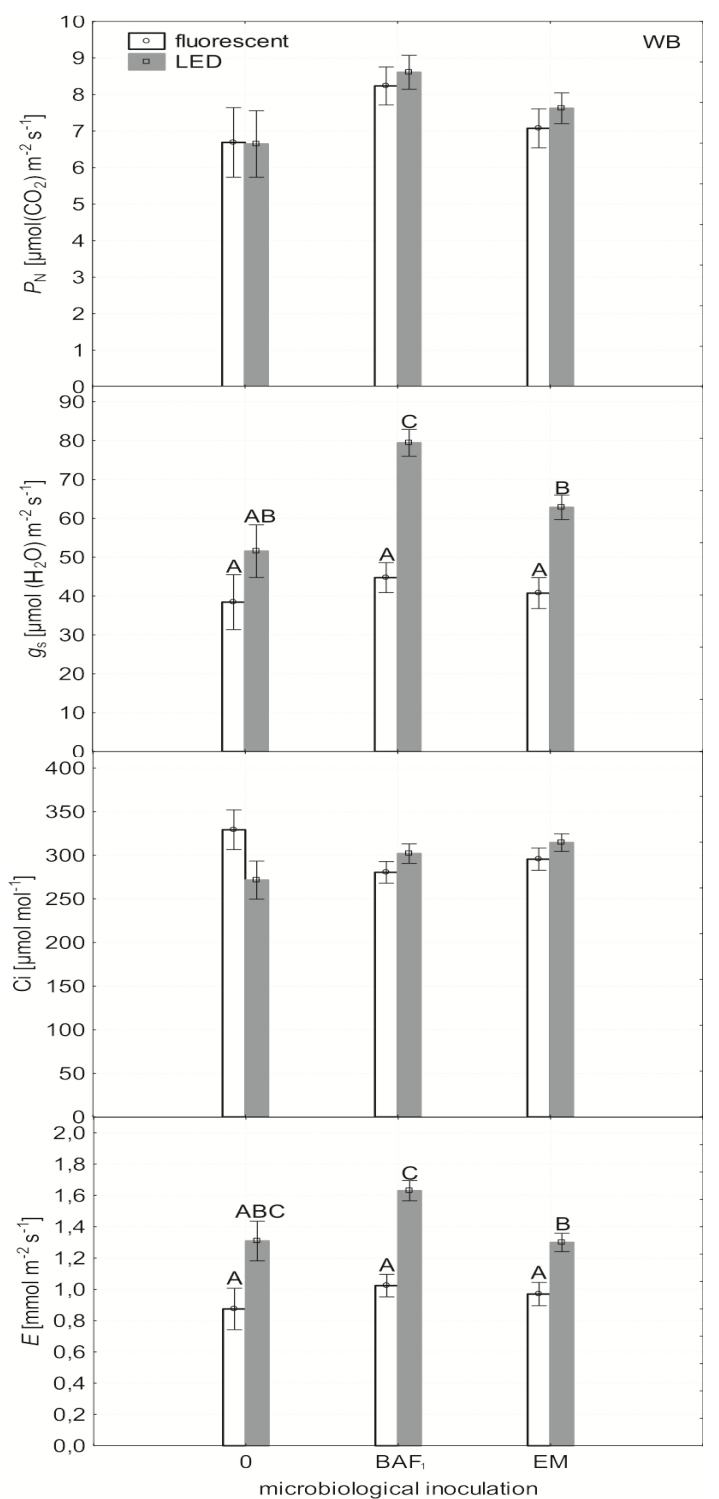

Fig. 3. Means $\pm \mathrm{SE}$ of net photosynthesis rate $(\mathrm{PN})$, stomatal conductance (gs), intercellular $\mathrm{CO} 2$ concentration $(\mathrm{Ci})$ and transpiration rate (E) of plants cultivated in white blue (WB) light colour conditions and treated by different microbiological inoculum (BAF1 - Bacteria-Actinomycetes-Fungi; EM - effective microorganisms). Letters denote significant differences between means at $\mathrm{p}<0.05$

\section{Results}

Two-way analysis of variance revealed no effect of lamp type on net photosynthetic rate and intercellular $\mathrm{CO}_{2}$ concentration in the case of white-blue light colour. There was also no effect of microbial inoculation on the latter parameter. However, both factors had a significant effect on both stomatal conductance and transpiration rate (Table 1). There was no significant difference between net photosynthetic rate of plants cultivated under fluorescent and LED lamps. However, we can observe $P_{\mathrm{N}}$ increment tendencies in plants grown with microbial inoculation. Moreover, higher $P_{\mathrm{N}}$ levels were noted for plants treated with $\mathrm{BAF}_{1}$ microorganisms. The increase of $P_{\mathrm{N}}$ was connected with stomatal opening in the case of LEDs. This was not observed in the case of fluorescent lamps, where

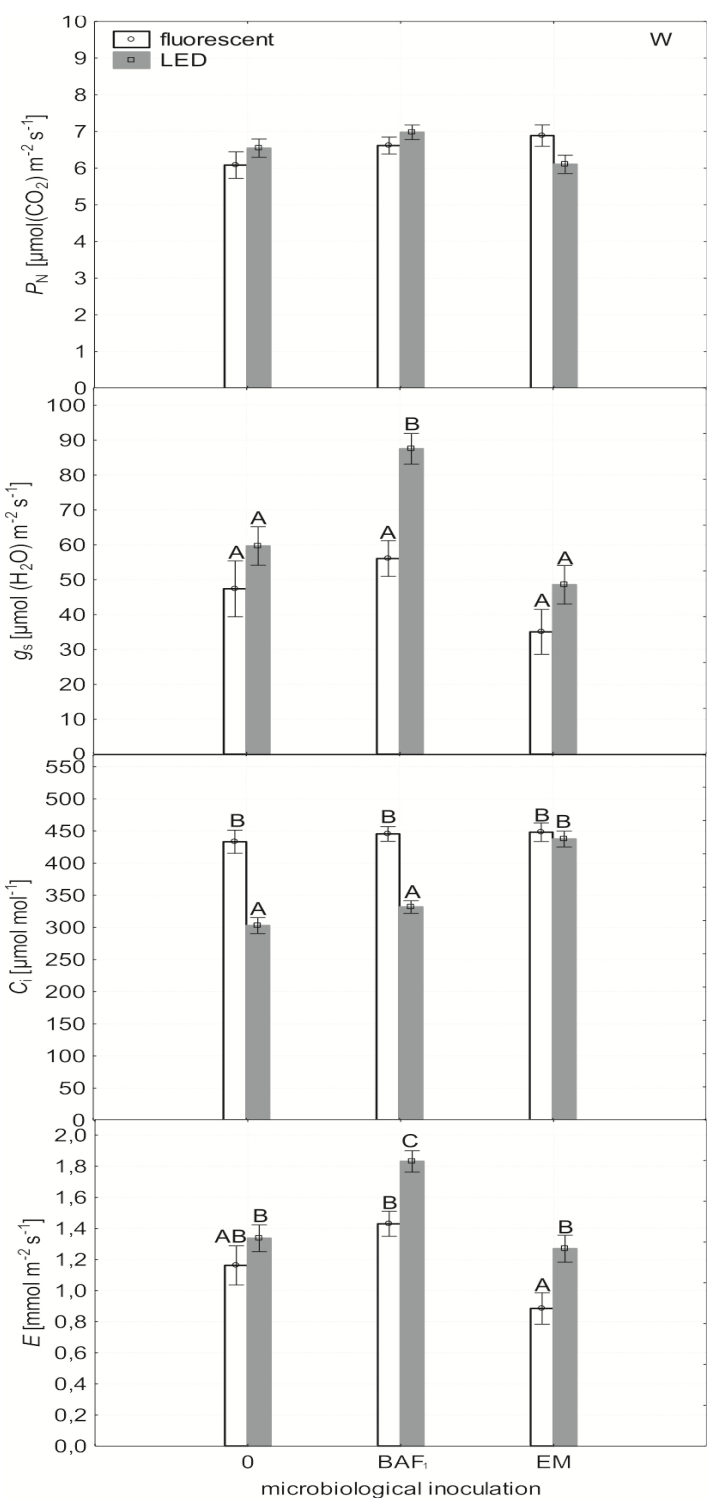

Fig. 4. Means \pm SE of net photosynthesis rate (PN), stomatal conductance (gs), intercellular $\mathrm{CO} 2$ concentration $(\mathrm{Ci})$ and transpiration rate (E) of plants cultivated in white (W) light colour conditions and treated by different microbiological inoculum (BAF1 - Bacteria-Actinomycetes-Fungi; EM effective microorganisms). Letters denote significant differences between means at $\mathrm{p}<0.05$ 
similar levels of $g_{s}$ were noted in both inoculation treatments as well as in control plants. Stomatal opening was also connected with transpiration rate; hence we can note higher values for plants cultivated under LEDs, for all three treatments. Although there are not (statistically significant) differences in the case of $C_{\mathrm{i}}$, higher levels were noted in the case of LEDs for both microbial treatments, while opposite results were note for plants under fluorescent lamp (Fig. 3).

There was no effect of lamp or inoculation type on net photosynthetic rate in the case of white light. However, a statistically significant influence of both factors was observed for stomatal conductance, intercellular $\mathrm{CO}_{2}$ concentration and transpiration rate (Table 1). There were no statistically significant differences between net photosynthetic rate of plants treated with

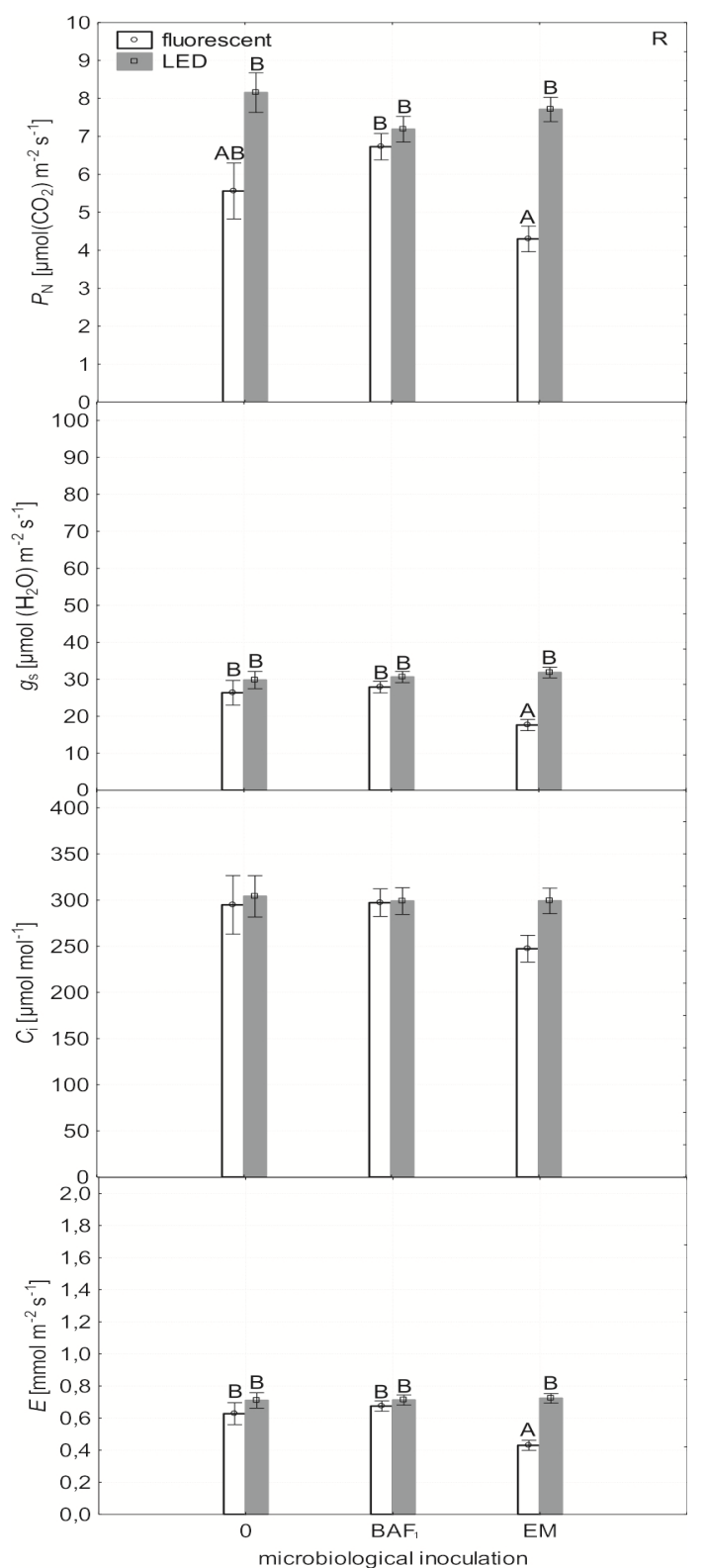

Fig. 5. Means $\pm \mathrm{SE}$ of net photosynthesis rate (PN), stomatal conductance (gs), intercellular $\mathrm{CO} 2$ concentration $(\mathrm{Ci})$ and transpiration rate $(\mathrm{E})$ of plants cultivated in red $(\mathrm{R})$ light colour conditions and treated by different microbiological inoculum (BAF1 Bacteria-Actinomycetes-Fungi; EM - effective microorganisms). Letters denote significant differences between means at $\mathrm{p}<0.05$ microorganisms or different lamp types. On the other hand higher stomatal conductance and transpiration rates under LEDs white light were observed, especially for $\mathrm{BAF}_{1}$ treatment. High $g_{\mathrm{s}}$ and $E$ were connected with low $C_{\mathrm{i}}$ (Fig. 4).

Red light statistically significantly influenced the net photosynthetic rate, stomatal conductance and transpiration rate (Table 1). Higher photosynthetic activity was noted in plants cultivated under LEDs for all three treatments, although not always statistically significant. Similar tendencies were noted for stomatal conductance, intercellular $\mathrm{CO}_{2}$ concentration and transpiration rate, although statistically significant differences between lamp types were recorded only for EM (Fig. 5).

No lamp type effect was found for blue-red light on net photosynthetic rate, or for microbial treatment on stomatal

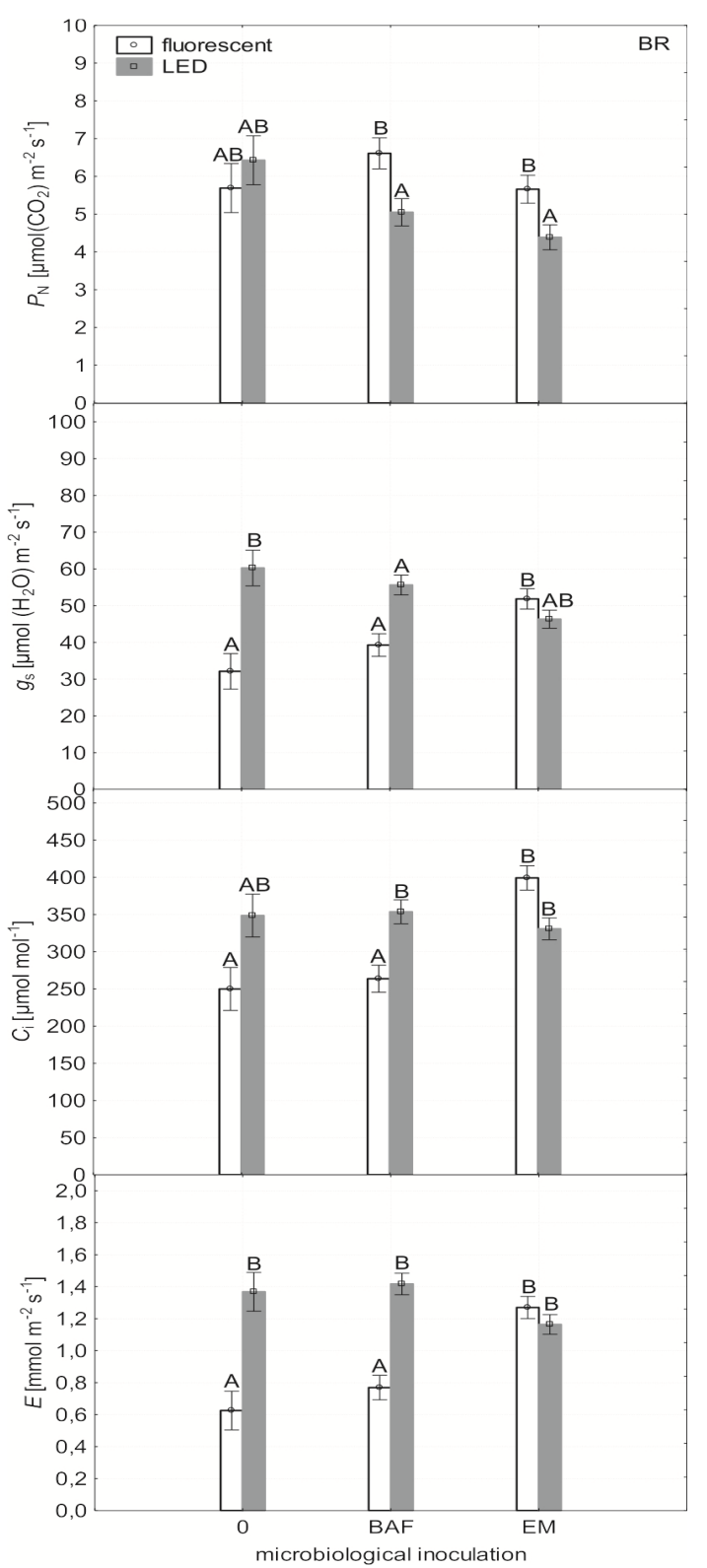

Fig. 6. Means $\pm \mathrm{SE}$ of net photosynthesis rate $\left(P_{\mathrm{N}}\right)$, stomatal conductance $\left(g_{\mathrm{s}}\right)$, intercellular $\mathrm{CO}_{2}$ concentration $\left(C_{\mathrm{i}}\right)$ and transpiration rate $(E)$ of plants cultivated in blue red (BR) light colour conditions and treated by different microbiological inoculum $\left(\mathrm{BAF}_{1}\right.$ Bacteria-Actinomycetes-Fungi; EM - effective microorganisms). Letters denote significant differences between means at $p<0.05$ 


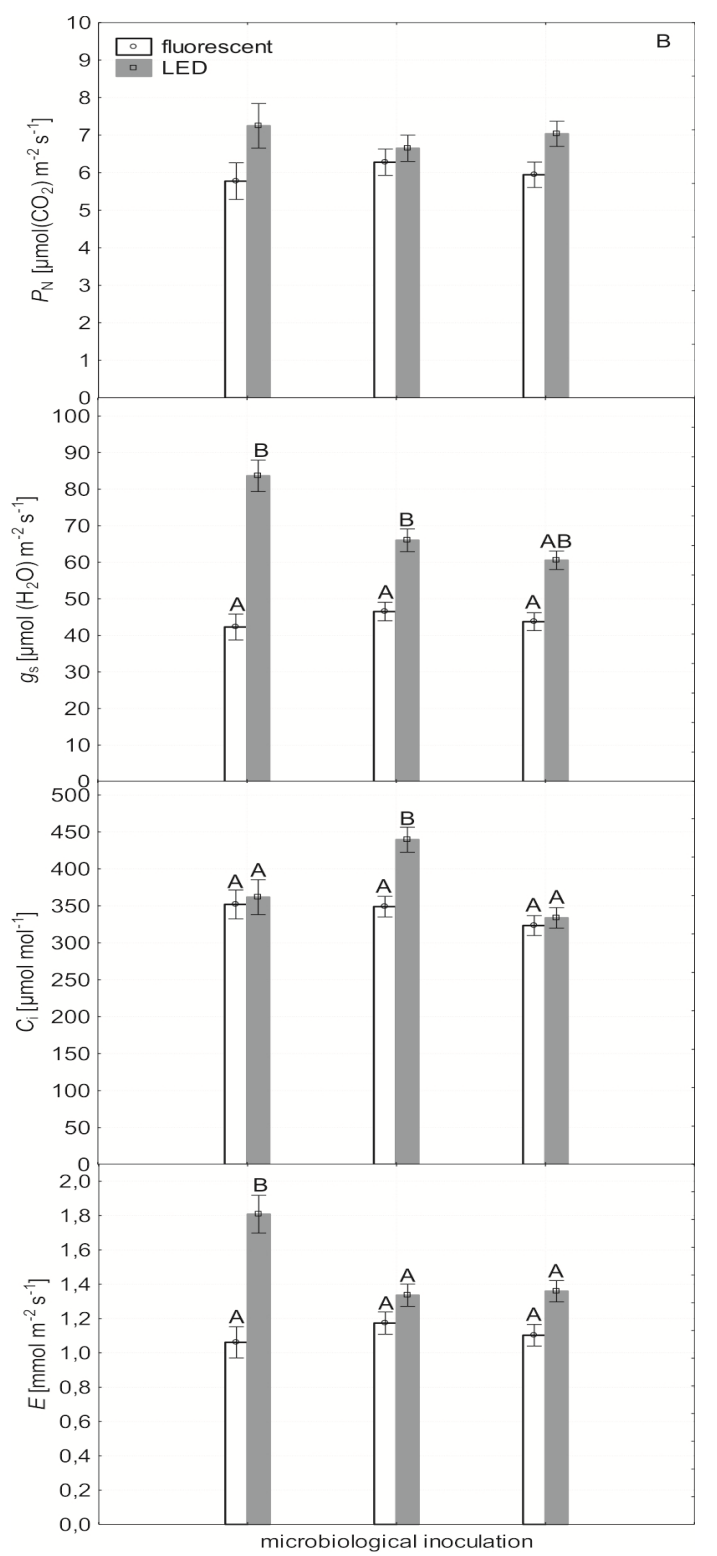

Fig. 7. Means $\pm \mathrm{SE}$ of net photosynthesis rate (PN), stomatal conductance (gs), intercellular $\mathrm{CO} 2$ concentration $(\mathrm{Ci})$ and transpiration rate (E) of plants cultivated in blue (B) light colour conditions and treated by different microbiological inoculum (BAF1 - Bacteria-Actinomycetes-Fungi; EM - effective microorganisms). Letters denote significant differences between means at $p<0.05$

conductance (Table 1). However, lower values of gas exchange parameters were noted in plants cultivated under LEDs and treated by microorganisms. While a positive effect of EM inoculation on $g_{s}, C_{\mathrm{i}}$ and $E$ was found only for plants cultivated under fluorescent lamps (Fig. 6).

Two-way ANOVA of the blue light effect revealed a statistically significant influence of lamp type and microbiological inoculation for all measured parameters, excluding the effect of microbial treatment factor on net photosynthetic rate (Table 1). Higher net photosynthetic rates were noted for plants cultivated under LEDs in all microbial treatments. Statistically significantly $(p<0.05)$ higher levels of stomatal conductance were observed in all treatments and especially in control plants. Similar observations were recorded for transpiration rate.

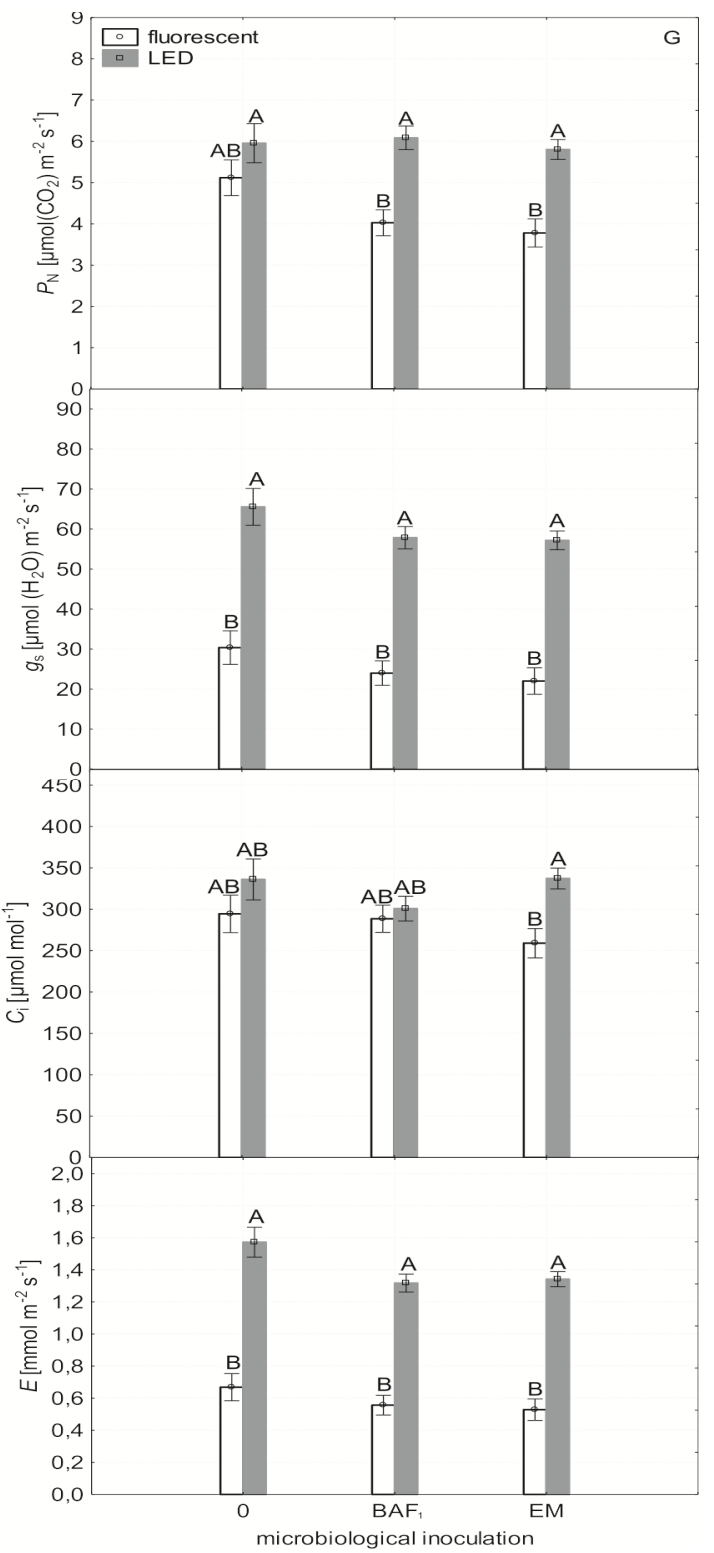

Fig. 8. Means $\pm \mathrm{SE}$ of net photosynthesis rate $(\mathrm{PN})$, stomatal conductance $(\mathrm{gs})$, intercellular $\mathrm{CO} 2$ concentration $(\mathrm{Ci})$ and transpiration rate (E) of plants cultivated in green (G) light colour conditions and treated by different microbiological inoculum (BAF1 Bacteria-Actinomycetes-Fungi; EM - effective microorganisms). Letters denote significant differences between means at $\mathrm{p}<0.05$

However, the effects on intercellular $\mathrm{CO}_{2}$ concentrations were not as clear as the other parameters, considering that $C_{\mathrm{i}}$ was increased only in $\mathrm{BAF}_{1}$ inoculation under blue LED light (Fig. 7).

Lamp type with green light influenced statistically significantly all measured parameters, while there was no such effect of microbial treatment (Table 1). Higher levels of all measured physiological parameters were observed for all plant treatments (control or inoculated) s grown under LED green light (Fig. 8). We can also observe a decrease of all measured physiological parameters in inoculated plants compared with the control ones under fluorescent green light.

The comparison of light quality was made based on control conditions without microbial treatment. The two-way 


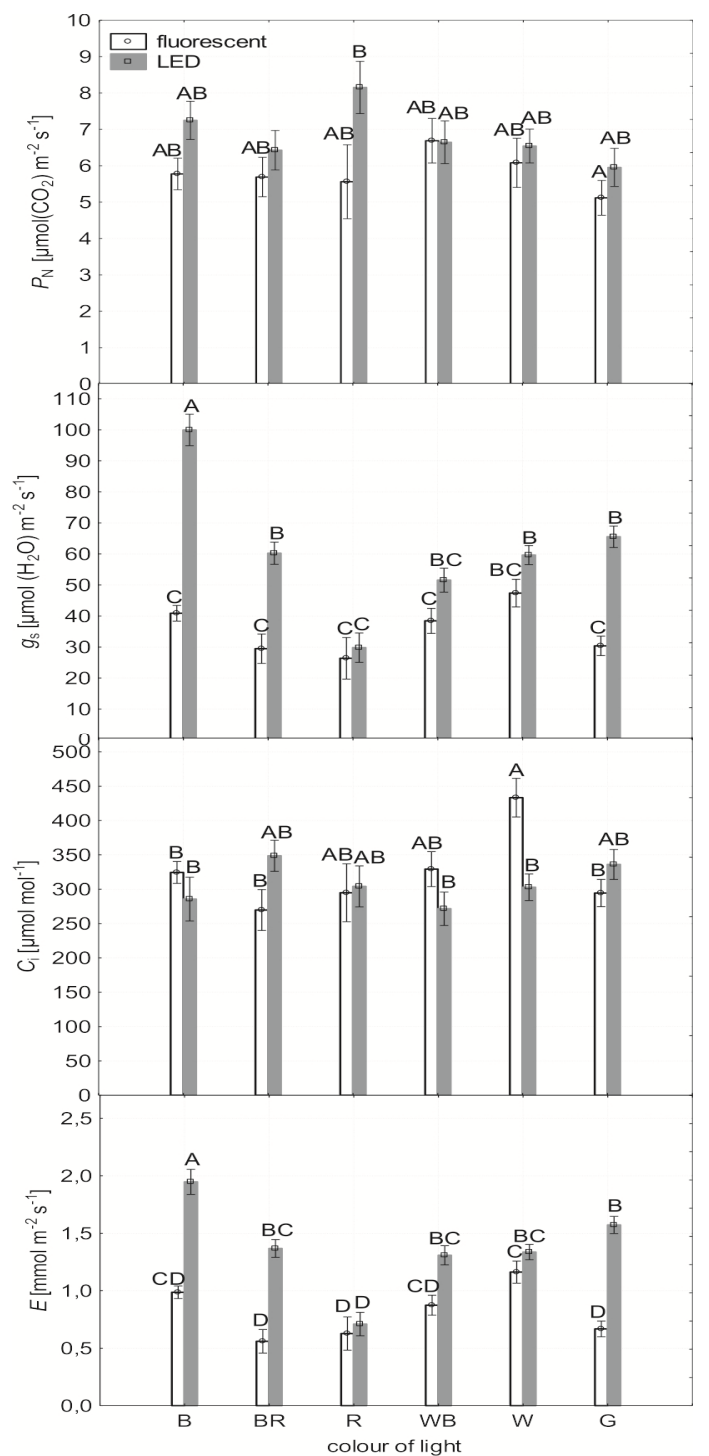

Fig. 9. Means $\pm \mathrm{SE}$ of net photosynthesis rate $\left(P_{\mathrm{N}}\right)$, stomatal conductance $\left(g_{s}\right)$, intercellular $\mathrm{CO}_{2}$ concentration $\left(C_{\mathrm{i}}\right)$ and transpiration rate $(E)$ of control plants (without microbiological inoculation) cultivated in different light colour conditions. Letters denote significant differences between means at $\mathrm{p}<0.05$

ANOVA revealed no statistically significant effect of lamp type or colour of light on net photosynthetic rate or intercellular $\mathrm{CO}_{2}$ concentration. A highly significant effect $(\mathrm{p}<0.001)$ was noted for stomatal conductance and transpiration rate (Table 2 ). The net photosynthetic rate was higher or at similar level (white-blue) in plants cultivated under LED. However, only for red and blue light the differences were statistically significant $(p<0.05)$. Higher photosynthesis activity was related to higher stomatal opening and transpiration rate (especially in the cases of blue and blue-red light) under LED lamps (Fig. 9).

\section{Discussion}

The artificial light plays an important role in plant production. The proper light quality can prolong good quality of plants, including longer and proper time of flowering. The proper balance of light conditions, medium properties and
Table 1. Two-way analysis of variance results (F-statistics and significance levels) of net photosynthetic rate (PN), stomatal conductance $(\mathrm{gs})$, intercellular $\mathrm{CO} 2$ concentration $(\mathrm{Ci})$ and transpiration rate $(\mathrm{E})$ of plants cultivated under different light colour conditions with both different lamp and microorganism types treatment impact factors

\begin{tabular}{|c|c|c|c|c|}
\hline $\begin{array}{l}\text { Colour } \\
\text { light }\end{array}$ & Parameter & $\begin{array}{l}\text { Lamp } \\
\text { type }\end{array}$ & $\begin{array}{l}\text { Microorganisms } \\
\text { treatment type }\end{array}$ & Interaction \\
\hline \multirow{4}{*}{$\begin{array}{c}\text { WB } \\
\text { (white } \\
\text { blue) }\end{array}$} & $P_{\mathrm{N}}$ & $0.3^{\text {ns }}$ & $3.9^{*}$ & $0.1^{\mathrm{ns}}$ \\
\hline & $g_{\mathrm{s}}$ & $32.9^{* *}$ & $6.6^{* * *}$ & $2.6^{\mathrm{ns}}$ \\
\hline & $C_{\mathrm{i}}$ & $0.2^{\mathrm{ns}}$ & $0.8^{\text {ns }}$ & $2.7^{\mathrm{ns}}$ \\
\hline & $E$ & $36.1^{* * *}$ & $5.0^{* *}$ & $2.1^{\mathrm{ns}}$ \\
\hline \multirow{4}{*}{$\begin{array}{c}\text { W } \\
\text { (white) }\end{array}$} & $P_{\mathrm{N}}$ & $0.1^{\mathrm{ns}}$ & $1.8^{\mathrm{ns}}$ & $3.3^{*}$ \\
\hline & $g_{\mathrm{s}}$ & $15.4^{* * *}$ & $16.1^{* * *}$ & $1.9^{\mathrm{ns}}$ \\
\hline & $C_{\mathrm{i}}$ & $60.9^{* * *}$ & $15.6^{* * *}$ & $11.6^{* * *}$ \\
\hline & $E$ & $17.6^{* * *}$ & $22.9^{* * *}$ & $0.8^{\mathrm{ns}}$ \\
\hline \multirow{4}{*}{$\begin{array}{c}\mathrm{R} \\
(\mathrm{red})\end{array}$} & $P_{\mathrm{N}}$ & $32.9^{* * *}$ & $4.4^{*}$ & $9.8^{* * *}$ \\
\hline & $g_{\mathrm{s}}$ & $15.9^{* * *}$ & $4.5^{*}$ & $7.8^{* * *}$ \\
\hline & $C_{\mathrm{i}}$ & $1.7^{\mathrm{ns}}$ & $1.7^{\mathrm{ns}}$ & $1.6^{\mathrm{ns}}$ \\
\hline & $E$ & $15.6^{* * *}$ & $7.3^{* *}$ & $8.8^{* * *}$ \\
\hline \multirow{4}{*}{$\begin{array}{c}\text { BR } \\
\text { (red } \\
\text { blue) }\end{array}$} & $P_{\mathrm{N}}$ & $3.2^{\mathrm{ns}}$ & $3.3^{*}$ & $2.4^{\mathrm{ns}}$ \\
\hline & $g_{\mathrm{s}}$ & $19.5^{* * *}$ & $0.3^{\mathrm{ns}}$ & $12.9^{* * *}$ \\
\hline & $C_{\mathrm{i}}$ & $5.2^{*}$ & $7.5^{* * *}$ & $13.9^{* * *}$ \\
\hline & $E$ & $34.0^{* * *}$ & $3.2^{*}$ & $18.7^{* * *}$ \\
\hline \multirow{4}{*}{$\begin{array}{c}\text { B } \\
\text { (blue) }\end{array}$} & $P_{\mathrm{N}}$ & $8.1^{* *}$ & $0.1^{\mathrm{ns}}$ & $0.9^{\mathrm{ns}}$ \\
\hline & $g_{\mathrm{s}}$ & $100.9^{* * *}$ & $5.5^{* *}$ & $7.3^{* * *}$ \\
\hline & $C_{\mathrm{i}}$ & $6.8^{* *}$ & $10.0^{* * *}$ & $4.3^{*}$ \\
\hline & $E$ & $36.9^{* * *}$ & $3.1^{*}$ & $6.1^{* *}$ \\
\hline \multirow{4}{*}{$\begin{array}{c}\mathrm{G} \\
\text { (green) }\end{array}$} & $P_{\mathrm{N}}$ & $31.4^{* * *}$ & $1.9^{\text {ns }}$ & $1.4^{\mathrm{ns}}$ \\
\hline & $g_{\mathrm{s}}$ & $150.2^{* * *}$ & $2.6^{\mathrm{ns}}$ & $0.1^{\mathrm{ns}}$ \\
\hline & $C_{\mathrm{i}}$ & $8.4^{* *}$ & $0.5^{\mathrm{ns}}$ & $2.2^{\mathrm{ns}}$ \\
\hline & $E$ & $208.1^{* * *}$ & $3.5^{*}$ & $0.4^{\mathrm{ns}}$ \\
\hline
\end{tabular}

Table 2. Two-way analysis of variance results (F-statistics and significance levels) of net photosynthetic rate $\left(P_{\mathrm{N}}\right)$, stomatal conductance $\left(g_{\mathrm{s}}\right)$, intercellular $\mathrm{CO}_{2}$ concentration $\left(C_{\mathrm{i}}\right)$ and transpiration rate $(E)$ of control plants (lack of microorganism applications - BAF or EM) with lamp colour and type impact factors

\begin{tabular}{cccc}
\hline Parameter & Colour & Lamp type & Iinteraction \\
\hline$P_{\mathrm{N}}$ & $2.1^{\mathrm{ns}}$ & $2.4^{\mathrm{ns}}$ & $1.0^{\mathrm{ns}}$ \\
$g_{\mathrm{s}}$ & $17.8^{* * *}$ & $108.7^{* * *}$ & $11.4^{* * *}$ \\
$C_{\mathrm{i}}$ & $2.2^{\mathrm{ns}}$ & $1.1^{\mathrm{ns}}$ & $4.7^{* * *}$ \\
$E$ & $13.7^{* * *}$ & $111.7^{* * *}$ & $8.8^{* * *}$ \\
\hline
\end{tabular}

${ }^{* * *} \alpha \leq 0,001 ;{ }^{* *} \alpha \leq 0,01 ;{ }^{*} \alpha \leq 0,05 ;$ ns - not significant

WB- white blue; W - white; R - red; BR - red blue; B - blue; G - green

nutrition can influence on plant quality and their economic value. Hence, it is very important to find adjust plant cultivation process and their storage for their life cycle and further functioning, e.g photosynthetic activity.

The presented investigations revealed the higher effect of lamp type on reached maximum photosynthetic activity plants cultivated in such conditions for few weeks.

Our results are in agreement with previous investigations on effects of blue light on stomatal opening, due to presence of chloroplasts in guard cells, as well as due to blue-light receptors phototropins (Wu 2008). However, there are many reports on the effect of monochromatic light on the photosynthetic process. Monochromatic light (except blue) mostly caused a decrease of 
32

$P_{\mathrm{N}}$ in comparison to plants cultivated under broad spectral light (Yu and Ong, 2003; Xiaoying et al., 2012). This fact was usually explained as an effect of the narrow transmission peaks, leading to an imbalance of photons available to PSI and PSII and thus changing the ratio of cyclic to whole chain electron transport (Tennessen et al., 1994). Concerning plants cultivated without microbial inoculation we observed lower $P_{\mathrm{N}}$ levels in plant cultivated under combined red-blue light in comparison to monochromatic red or blue light (Fig. 9). Several authors have reported a higher level of photosynthesis in plants cultivated under blue light as well as combinations of blue with other colours of light in many plant species (Lee et al., 2011; Xiaoying et al., 2012). On the other hand, Kim et al. (2004b) found a lower net photosynthetic rate in chrysanthemum cultivated under blue in comparison to red LEDs, and moreover to fluorescent lamps. However, they also observed the highest activity of photosynthesis in plants cultivated under blue-red LEDs, and a more consistent chlorophyll absorption for these two colours was suggested as the reason for this situation.. In our results, concerning plants without microbial inoculation, similar levels of stomatal conductance in red-blue and white light were noted, while Kim et al. (2004a) found lower values of stomatal opening in plants cultivated under combination in comparison to monochromatic lamp. However, these were not correlated with further plant growth. While Karlsson et al (1986) found no effect of blue light on guard cells and stomatal opening. There are many uncertainties for proper combination of blue-red light for certain plant species. Considering plants without microbial inoculation and based on our results it seems that Pelargonium zonale revealed adaptation properties and reach the highest maximum $P_{\mathrm{N}}$ level after few weeks cultivation under red LEDs. Moreover, the lowest stomatal conductance and transpiration rate was also noted in plants under red light of both lamp types.

Considering, plants cultivated in the medium with and without microbial inoculation, highest maximum of $P_{\mathrm{N}}$ values were noted in plants cultivated under a mixture of white-blue lights (both - fluorescent and LED) with $\mathrm{BAF}_{1}$ inoculation (Fig. 3). In the case of white light the treatment with $\mathrm{BAF}_{1}$ microorganisms influenced stomatal conductance and transpiration rate (Fig. 4), probably due to maintenance of the physiological balance (Martin and Botton, 1993; Smith and Read, 2008). Xu et al. (2008) found that maize stomata of nonwatered plants treated by EM respond more quickly and efficiently in comparison to plants well-watered and without EM. This can be an indirect beneficial of microorganisms on production of some phytohormones, and enhance of growth, development and further plant response efficiency to stress factors. The stomatal conductance increase due to EM application was also reported by Chantal et al. (2010), while transpiration rate differed much more between various EM application and $\mathrm{Ci}$ did not differ. The last one was also noted in our investigations. The increase of stomatal conductance in the case of red light may be connected with a favourable light spectrum (Fig. 9), as well as with increase of nutrient uptake caused by microorganisms (Martin et al., 2001; Smith and Read, 2008). The comparable maximum $P_{\mathrm{N}}$ level was observed in both microorganisms inoculation for all lamp types to plants without microbial inoculation. This is in agreement with previous investigations, which indicated on positive role for photosynthetic activity due to continual supply of nutrient and application together with EM phytohormones and other biologically active substances which can delay the process of senescence and increase photosynthesis (Yamada and Xu 2000). The last aspect can also have a positive effect for proper storage of ornamental plants and prolonged possibilities for trading of good quality of plants. The better soil nutrient transformation, higher root transformation, higher uptake of nutrients and their movement in plants and as a result higher photosynthetic activity was previously proved in various investigations (Okorski et al. 2010; El-Shatnawi and Makhadmeh, 2001). Our investigations, proved their role for ornamental plants cultivated under different light conditions.

\section{Conclusion}

Our investigations revealed that the highest maximum of $P_{\mathrm{N}}$ revealed plants cultivated several weeks under white-blue light LED, fluorescent and LED, and inoculated by BAF. Moreover, these plants also were found with the higher stomatal conductance and transpiration rate in comparison to control plants without $\mathrm{BAF}_{1}$. Considering plants without microbial inoculation, the maximum level of $P_{\mathrm{N}}$ reached plants after cultivation under red LED. Overall, we can conclude that adaptation to long-term light quality is not dependent on microbial inoculation, as small differences between gas exchange parameters were observed among non-inoculated and inoculated plants in certain light colours. The positive effect of EM was only noted in the case of plants cultivated under blue-red fluorescent light.

\section{Acknowledgments}

The study was support by Ministry of Science and Higher Education grant no. NN 310444938.

\section{References}

Borkowska B (2002). Growth and photosynthesis activity of micropropagated strawberry plants inoculated with endomycorrhizal fungi (AMF) and growing under drought stress. Acta Physiologiae Plantarum 24:365-370.

Chantal K, Xiaohou S, Weimu W, Iro Ongor BT (2010). Effects of effective microorganisms on yield and quality of vegetable cabbage comparatively to nitrogen and phosphorus fertilizers. Pakistan Journal of Nutrition 9(11):1039-1042.

Domurath N, Schroeder FG, Glatzel S (2012). Light response curves of selected plants under different light conditions. Acta Horticulturae 956:291-298.

El-Shatnawi MKJ, Makhadmeh IM (2001). Ecophysiology of the plantrhizospheresystem. Journal of Agronomy and Crop Science 187(1):1-9.

Goins GD, Yorio NC, Sanwo MM, Brown CS (1997). Photomorphogenesis, photosynthesis, and seed yield of wheat plants grown under red light-emitting diodes (LEDs) with and without supplemental blue lighting. Journal of Experimental Botany 48(7):1407-1413.

Golovatskaya IF (2005). The role of cryptochrome 1 and phytochromes in the control of plant photomorphogenetic responses to green light. Russian Journal of Plant Physiology 52(6):724730.

Goto E (2012). Plant production in a closed plant factory with artificial lighting. Acta Horticulturae 956:37-50. 
Higa T (1991). Effective microorganism: a biotechnology for mankind. In: Parr JF, Hornick SB, Whitman CE (Eds). Proc First International Conference on Kyusei Nature Farming. US Department of Agriculture, Washington DC pp 118-124.

Jerzy M, Zakrzewski P, Schroeter-Zakrzewska A (2011). Effect of light on the opening of inflorescence buds and post-harvest longevity of pot chrysanthemum (Chrysanthemum $\times$ grandiflorum (Ramat.) Kitam.). Acta Agrobotanica 64(3):13-18.

Karlsson PE (1986). Blue light regulation of stomata in wheat seedlings. II. Action spectrum and search for action dichroism. Physiologia Plantarum 66(2):207-210.

Kim HH, Goins GD, Wheeler RM, Sager JC (2004). Stomatal conductance of lettuce grown under or exposed to different light qualities. Annals of Botany 94(5):691-697.

Kim SJ, Hahn EJ, Heo JW, Paek KY (2004). Effects of LEDs on net photosynthesis rate, growth and leaf stomata of chrystantemum plantlets in vitro. Scientia Horticulturae 101(1):143-151.

Kozai T, Ohyama K, Chun C (2006). Commercialized closed systems with artificial lighting for plant production. Acta Horticulturae 71:61-67.

Lee YI, Fang W, Chen CC (2011). Effect of six different LED light qualities on the seedlings growth of Paphiopedilum orchid in vitro. Acta Horticulturae 907:389-392.

Liu XY, Chang TT, Guo SR, Xu ZG, Li J (2011). Effect of different light quality of LED on growth and photosynthesic character in cherry tomato seedlings. Acta Horticulturae 907:325-330.

Marschner P (2007). Plant-microbe interactions in the rhizosphere and nutrient cycling. In: Marsner P, Rengel Z (Eds). Nutrient Cyding in Terrestrial Ecosystems, Soil Biology.Springer-Verlag, Berlin pp 159-182.

Martin F, Botton B (1993). Nitrogen metabolism of ectomycorrhizal fungi and ectomycorrhizas. Advances in Plant Pathology 9:83-102.

Martin F, Cliquet J-B, Stewart G (2001). Nitrogen acquisition and assimilation in mycorrhizal symbioses. In: Lea PJ, Morot-Gaudry JF (Eds). Plant Nitrogen.Springer-Verlag, Berlin pp 147-167.

Matsuda R, Ohashi-Kaneko K, Fujiwara K, Kurata K (2008). Effects of blue light deficiency on acclimation of light energy partitioning in PSII and $\mathrm{CO}_{2}$ assimilation capacity to high irradiance in spinach leaves. Plant and Cell Physiology 49(4):664-670.

McMahon MJ, Kelly JW, Decoteau R, Young RE, Pollock RK (1991). Growth of Dendranthema grandiflorum (Ramat) Kitamura under various spectral filters. Journal of the American Society for Horticultural Science 116(6):950-954.

Morrow RC (2008). LED lighting in horticulture. HortScience 43(7):19471950.

Nowak J (2004). Effects of arbuscular mycorrhizal fungi and organic fertilization on growth, lowering, nutrient uptake, photosynthesis and transpiration of geranium (Pelangonium hortorum L.H. Bailey ${ }^{\circ}$ Tango Orange').Symbiosis 37:259-266.

Okorski A, Olszewski J, Głowacka K, Okorkka S, Pszczółkowska A (2010). The effect of the application of the biological control agent eml on gas exchange parameters and productivity of Pisum sativum $\mathrm{L}$. infected with fusarium Oxysporum schlecht. Acta Agrobotanica 63(2):105-115.

Reinders U, Dueck TA (2008). LEDs are still the future of lighting. FlowerTECH.11(6):2426.
Sxbø A, Krekling T, Appelgren M (1995). Light quality affects photosynthesis and leaf anatomy of birch plantlets in vitro. Plant Cell, TissueandOrgan Culture 41(2):177-185.

Schuerger AC, Brown CS, Stryjewski EC (1997). Anatomical features of pepper plants (Capsicum annuum L.) grown under red light-emitting diodes supplemented with blue or far-red light. Annals of Botany 79(3):273-282.

Senger H (1982). The effect of blue light on plants and microorganisms. Photochemistryand Photobiology35(6):911-920.

Smith SE, Read D (2008). Mycorrhizal symbiosis (3th ed). Academic Press,London, UK.

Stielow G (2003). Rich soil do not need of the fertilization. Journal of Research and Applications in Agricultural Engineering 48(1):20-22.

Sabzalian MR, Heydarizadeh P, Zahedi M, Boroomand M, Agharokh M, Sahba MR, Schoefs B (2014). High performance of vegetables, flowers, and medicinal plants in a red-blue LED incubator for indoor plant production. Agronomy for SustainableDevelopment 34(4):879-886.

Tanaka M, Takamura T, Watanabe H, Endo M, Yanagi T, Okamoto K (1998). In vitro growth of Cymbidium plantlets cultured under superbright red and blue light-emitting diodes (LEDs). The Journal of Horticultural Science and Biotechnology 73(1):39-44.

Tennessen DJ, Singsaas EL, Sharkey TD (1994). Light-emitting diodes as a light source for photosynthesis research. Photosynthesis Research 39(1):85-92.

Voskresenskaya NP (1987). Photoregulatory reactions and activity of the photosyntheticapparatus. SovietPlant Physiology34(4):538-550.

Wolna-Maruwka A,Schroeter-Zakrzewska A, BorowiakK, NiewiadomskaA (2014). Analysis of the effect of BAF1 microbial inoculum on microbiological and biochemical condition of peat under scarlet sage cultivation. Fresenius Environmental Bulletin 23(1a):228-238.

Wolna-Maruwka A, Mocek-Płóciniak A, Schroeter-Zakrzewska A, Niewiadomska A, Piechota T, Swędrzyńska D, Kosicka D, Pilarska A (2015). An analysis of the influence of a microbial preparation on the enzymatic parameters of peat and morphological traits of the french marigold.Nauka Przyroda Technologia 9(4):47:1-14.

Wu Y (2008). Guard cell signaling. Annual Plant Reviews. Intracellular Signalingin Plants 33:362-387.

XiaoyingL, ShirongG, Taotao C,ZhigangX, Tezuka T (2012). Regulation of the growth and photosynthesis of cherry tomato seedlings by different light irradiations of light emitting diodes (LED). African Journal of Biotechnology 11(22):6169-6177.

XuH-L, WangX-, WangJ (2008). Effect of a microbial inoculant on stomatal response of maizeleaves.Journal ofCrop Production 3(1):235-243.

Yamada K, Xu H (2000). Properties and applications of an organic fertilizer inoculated with Effective Microorganisms. In: Xu H, Parr JF, Umemura $\mathrm{H}$ (Eds). Nature farming and microbial applications. New York pp 255268.

Yu H, Ong BL (2003). Effect of radiation quality on growth and photosynthesis of Acacia mangium seedlings. Photosynthetica 41:349355.

Zeiger E, Talbott LD, FrechillaS, Srivastava A, Zhu JX (2002). The guard cell chloroplast: a perspective for the twenty-first century. New Phytologist 153(3):415-424. 